\title{
3D Nano-Probes for Thermal Microscopy
}

Jürgen Sattelkow ${ }^{1}$, Johannes E. Froech ${ }^{1}$, Robert Winkler ${ }^{1,2}$, Christian Schwalb ${ }^{3}$, Marcel Winhold ${ }^{3}$, Ernest G. Fantner ${ }^{3}$, Harald Plank ${ }^{1,2}$

1. Institute of Electron Microscopy and Nanoanalysis, Graz University of Technology, Graz, Austria.

2. Graz Centre for Electron Microscopy, Graz, Austria.

3. GETec Microscopy Inc. \& SCL Sensor.Tech. Fabrication Inc., Vienna, Austria.

With the recent introduction of controlled 3D nano-printing via focused electron beam induced deposition (FEBID) [1,2,3], entirely new ranges of applications such as nano-optics [4], -sensors [5], magnetics [6] or -mechanics comes within reach whose fabrication is extremely challenging or even impossible with alternative techniques. In this study, we use 3D FEBID for the direct-write fabrication of thermal 3D nano-probes for further application in Scanning Thermal Microscopy (SThM). In more detail, freestanding Pt-C multi-branch architectures are used as thermistors, where the electric bridge resistivity changes in dependency on local temperatures during SThM. The main advantage of this nanobridge concept is the small volume in the tip merging zone together with end radii down to $5 \mathrm{~nm}$, which enable fast thermal response and high lateral resolution, respectively.

First, the relations between multi-branch design and nano-mechanical properties are studied by a combined approach of finite element simulations, Atomic Force Microscopy (AFM) based force spectroscopy and real-time imaging via scanning electron microscopy. This not only leads to identification of ideal overall geometries but also reveals the high demands on fabrication accuracy to minimize unwanted morphological twisting (see Figure 1) and non-linear mechanical behavior under force load. In the second step, we introduce a material tuning approach via post-growth e-beam curing, which effectively transforms the carbon matrix around the Pt nano-grains into $\mathrm{sp}^{3}$ hybridized $3 \mathrm{D}$ carbon networks [5]. This not only increases the overall mechanical stability but in particular improves the wear resistance of the tip apex for stable AFM imaging. In the final step, we use FEBIDs direct-write capabilities to modify pre-structured AFM cantilevers (see Figure 2) by fully optimized 3D nano-probes for active thermistor operation via monitoring the electric current through the 3D nano-bridges. Thermal response studies in ambient and vacuum conditions are presented and demonstrate temperature sensitivities with sub-degree resolution as well as response times better than $32 \mathrm{~ms} / \mathrm{K}$ for dynamic thermo-studies.

References:

[1] R Winkler et al, ACS Appl. Nano Mater. (2018), in press.

[2] JD Fowlkes et al, ACS Appl. Nano Mater. (2018), in press.

[3] JD Fowlkes et al, ACS Nano 10 (2016), p. 6163.

[4] R Winkler et al, ACS Appl. Mater. Interfaces 9 (2017), p. 8233.

[5] G Arnold et al, Adv. Funct. Mater. (2018), in press.

[6] M Al Mamoori et al, Materials (Basel) 11 (2018), p. 289. 

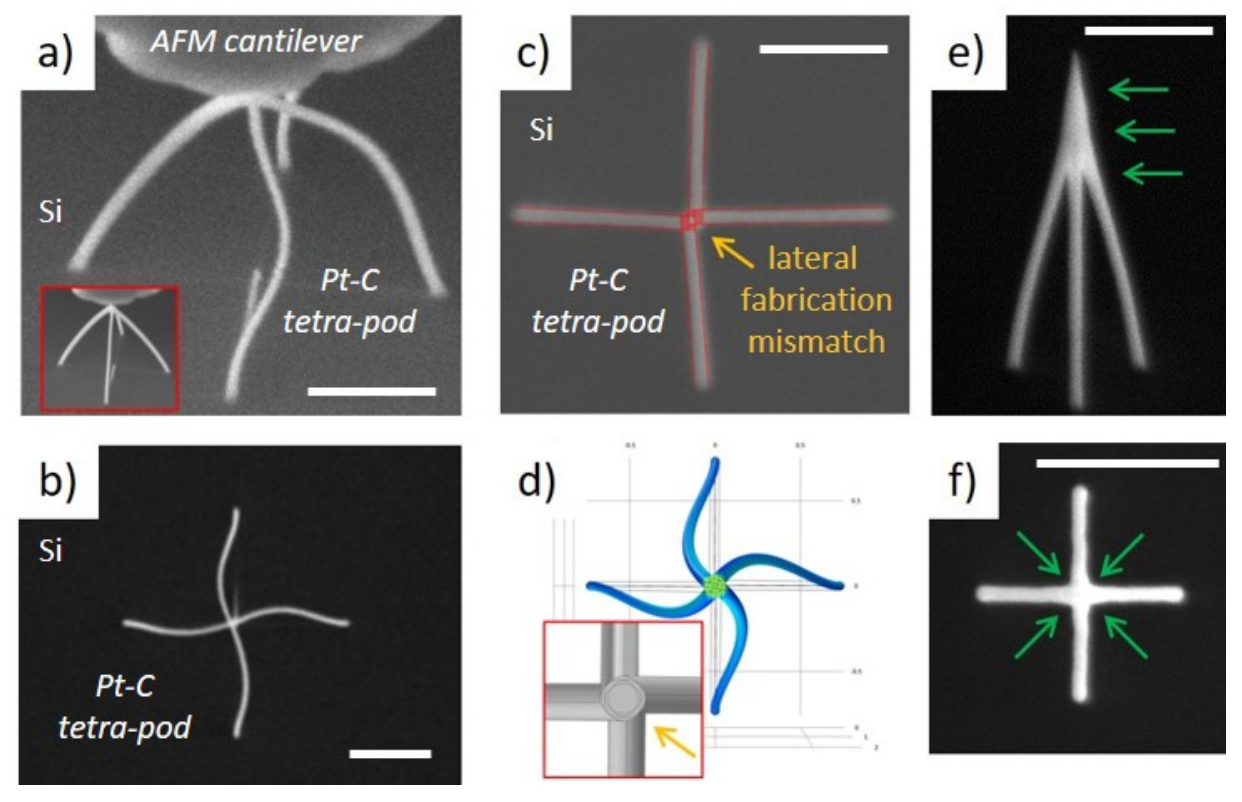

Figure 1. (a) tilted SEM image of a FEBID based Pt-C tetra-pod under compression using a premodified AFM cantilever. Compared to the initial shape (inset), morphological twisting is evident (see also top view in b). Closer inspection of such twisting tetra-pods reveal slight lateral mismatch in the central merging zone as evident in (c) by a top view SEM image before compression. (d) shows the result of a finite element simulation using real displacements (see inset). As evident, the simulations can clearly mimic the twisting behavior (compare $b$ and $d$ ) and identify such small mismatches as origin of unwanted morphological twisting. Together with further results on branch curvatures and general geometrical consideration, ideal tetra-pod architectures could be derived as shown in (e) and (f) by SEM side and top views. The main modification concerns an increasing merging zone on top (green arrows), which minimizes twisting effects even under high force load. SEM scale bars are $500 \mathrm{~nm}$.
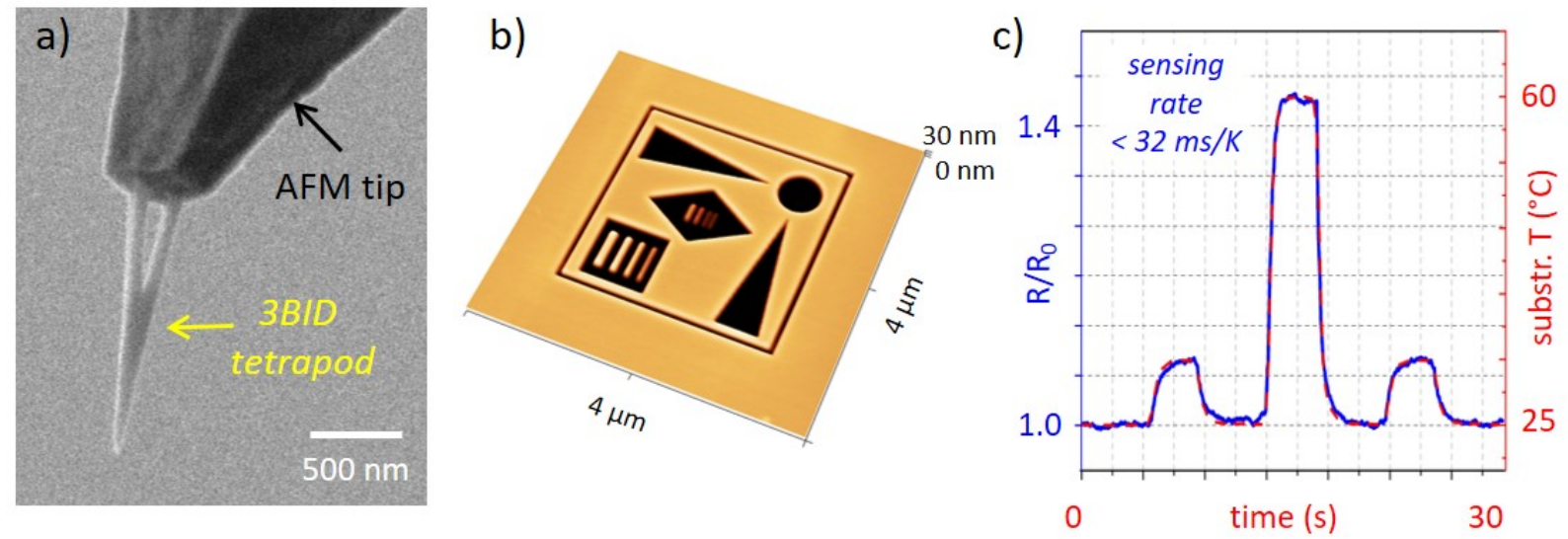

Figure 2. (a) optimized 3D tetra-pod fabricated on top of a pre-processed self-sensing cantilever. (b) shows the basic AFM performance on a FIB processed test structure, revealing lateral resolution in the sub-10 nm regime. (c) shows the timely response of such thermal 3D nano-probes by electric current readout through the $3 \mathrm{D}$ nano-bridges revealing sensing rates of $32 \mathrm{~ms} / \mathrm{K}$. 